\title{
Birth of fertile bimaternal offspring following intracytoplasmic injection of parthenogenetic haploid embryonic stem cells
}

Cell Research (2016) 26:135-138. doi:10.1038/cr.2015.151; published online 18 December 2015

\section{Dear Editor,}

Normal mammalian development requires participation of both maternal and paternal genomes because of the existence of genomic imprinting, whereas the gynogenetic and androgenetic embryos die shortly after implantation [1-3]. Generation of gynogenetic bimaternal mice containing two sets of maternal genomes was achieved using non-growing oocytes with imprinting modifications [4]; however, the approach was technically challenging and impractical for further applications. Recently, we and others have derived mammalian androgenetic and parthenogenetic haploid embryonic stem cells (ahESCs and phESCs), and showed that ahESCs can replace gametes to produce offspring [5-7], which provided alternative resources for reproduction $[6,7]$. Here we report that after proper imprinting modifications, the mouse phESCs can efficiently produce viable fertile offspring upon intracytoplasmic injection into MII oocytes. We thus establish a novel strategy of generating bimaternal mammals, which is valuable to uncover the function of genomic imprinting, and to improve assisted reproduction in diverse mammalian species.

Since our previous study showed that the MII oocyte-derived phESCs lost maternal imprinting in differentially methylated regions (DMRs) during culture [8], we hypothesized that bimaternal embryos generated by injecting the haploid phESCs into MII oocytes would have improved developmental potential compared with parthenogenetic embryos, which is known to die before embryonic day 9.5 (E9.5). To investigate the potential of phESCs to produce bimaternal mice, we first established phESC lines from mouse haploid parthenogenetic blastocysts carrying a constitutively expressed green fluorescent protein (Gfp) gene (Supplementary information, Figure S1A). The haploidy of phESC lines could be maintained by fluorescence-activated cell sorting (FACS)-based purification every 4-6 passages (Supplementary information, Figure S1B-S1D). The phESC lines expressed typical pluripotency marker genes (Supplementary information, Figure S1E), formed teratomas containing all three germ layers (Supplementary information, Figure S1F), and produced chimeric mice (Supplementary information, Figure S1G), indicating that they were pluripotent. Consistent with our hypothesis, the phESC-derived bimaternal embryos could develop beyond E10.5, but none developed beyond E13.5 (Supplementary information, Table S1A). One live E13.5 phESC-derived bimaternal fetus was generated (Figure $1 \mathrm{~A}$ and Supplementary information, Figure $\mathrm{S} 1 \mathrm{H})$. The reduced representation bisulfite sequencing (RRBS) analysis revealed that the maternally methylated DMRs showed similar methylation levels between wild-type (WT) embryos and the phESC-derived bimaternal embryo, but all 3 paternally methylated DMRs (H19-, IGand Rasgrf1-DMRs) were significantly hypomethylated in the bimaternal embryo compared with WT embryos (Figure 1B). Consistently, transcriptome analysis showed that only 8 of all imprinted genes had more than 2-fold difference of expression levels, including the upregulation of maternally expressed H19 and Rian, and the downregulation of paternally expressed $\operatorname{Ig} f 2$ and $\operatorname{Ig} f 2 a \mathrm{~s}$ in the bimaternal embryo; although the expression level of Rasgrf1 was very low at E13.5, it was also downregulated in the bimaternal embryo (Figure 1C and 1D). The global gene expression profiles exhibited high correlation $(\mathrm{R} 2=0.94)$ between the bimaternal embryo and WT control (Figure 1C). These results suggest that the aberrant expression of imprinted genes within the 3 paternally methylated imprinted clusters, H19-Igf2, Dlk1-Dio3 and Rasgrf1, likely contributed to the developmental defects of phESC-derived bimaternal embryos.

To improve the development of phESC-produced gynogenetic embryos, we modified the imprinting status of the H19-Igf2 and Dlk1-Dio3 clusters that controlled fetal growth by knocking out the DMRs, which may cause bidirectional loss of imprinting of all genes within the clusters. According to previous studies [9-13], three 
A

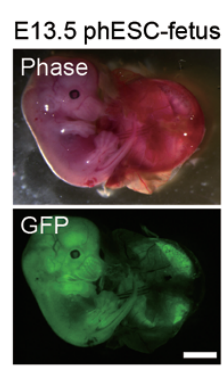

B

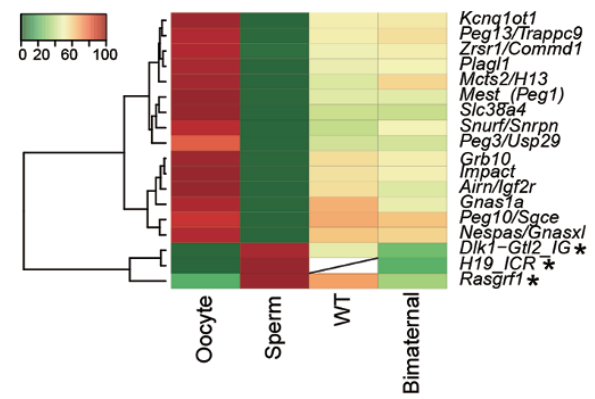

D

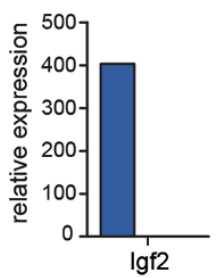

F

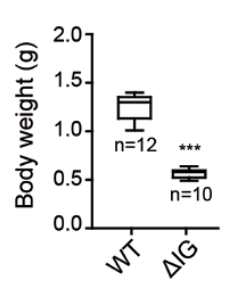

H

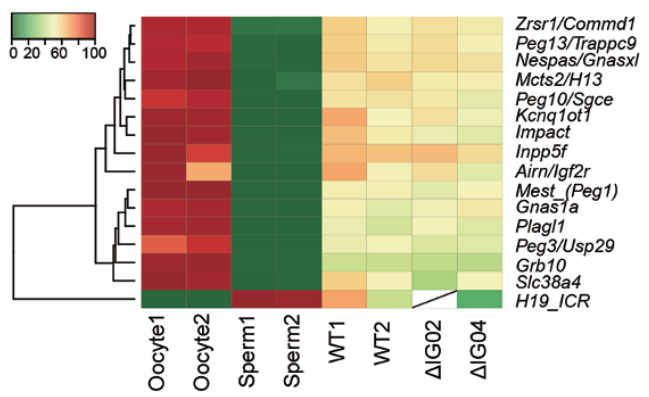

G
C
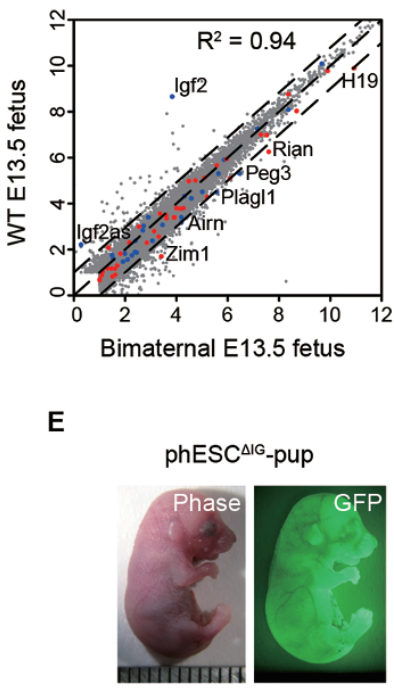
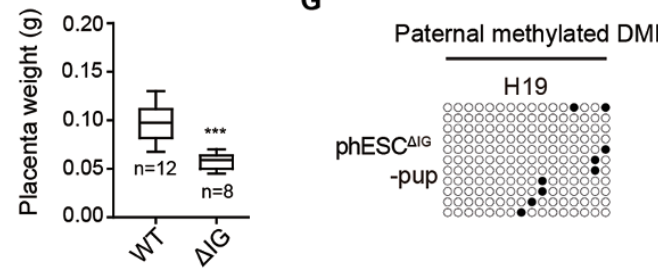

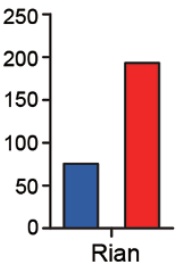

E

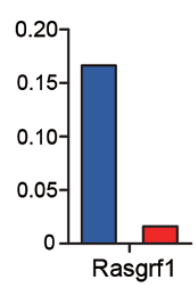

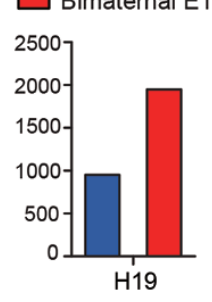

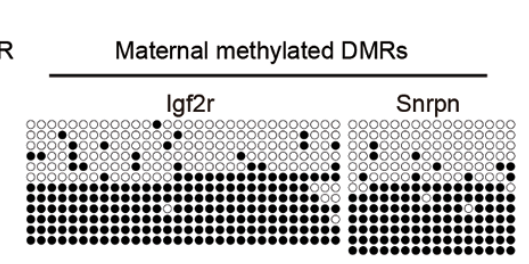

J
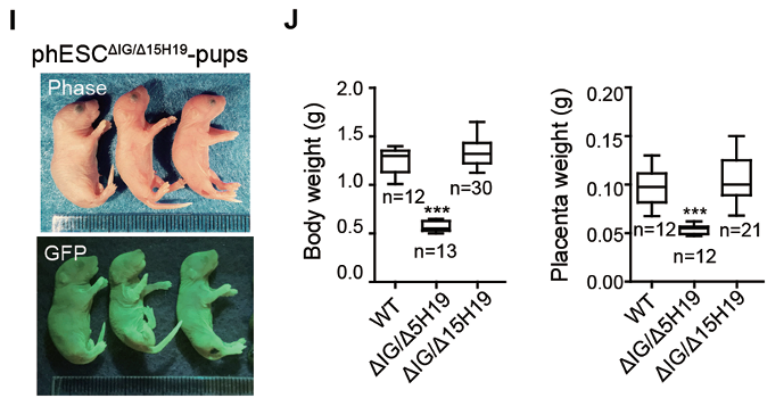

K

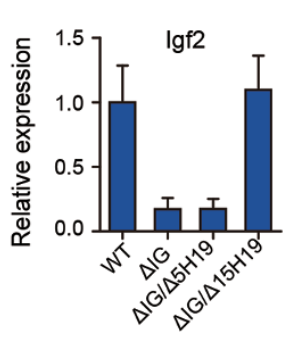

$\mathbf{L}$
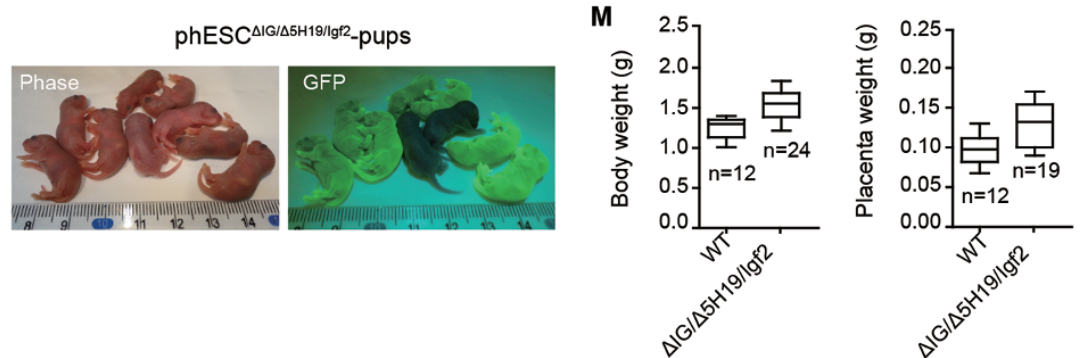

0

P

Q
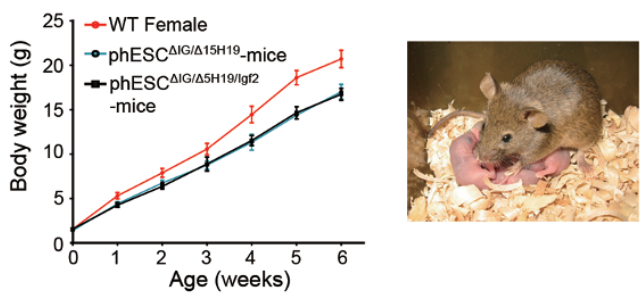

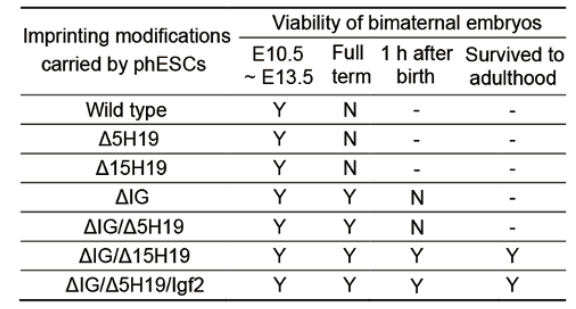


types of genetic deletions were designed, including a $\sim 5$ $\mathrm{kb}$ deletion of the H19-Igf2 locus (from $-4 \mathrm{~kb}$ to $+1 \mathrm{~kb}$ of $H 19$ transcription start site (TSS)), a $\sim 15 \mathrm{~kb}$ deletion (from $-10 \mathrm{~kb}$ to $+4 \mathrm{~kb}$ of H19 TSS) spanning the H19 gene, H19-ICR (from $-4 \mathrm{~kb}$ to $-2 \mathrm{~kb}$ of H19 TSS) and the upstream region of $H 19$-ICR, and a $\sim 4 \mathrm{~kb}$ deletion spanning the IG-DMR of the Dlk1-Dio3 cluster (Supplementary information, Figure S2A and S2E). To boost the efficiency of homologous recombination-mediated genetic knockout, we used the CRISPR/Cas9 system to introduce a targeted DNA double strand break in the knockout region. The correctly targeted clones were identified by PCR with specific primer pairs that could distinguish the WT and knockout alleles (Supplementary information, Figure S2B, S2F and Table S1C). Consistent with the genetic deletions, $H 19$ expression was abolished in phESCs carrying the $5 \mathrm{~kb}$ deletion $\left(\mathrm{phESC}^{\Delta 5 \mathrm{H} 19}\right.$ ) or the $15 \mathrm{~kb}$ deletion (phESC ${ }^{\Delta 15 H 19}$ ) of the H19-Igf2 locus, and Gt12 expression was silenced in phESCs carrying the IGDMR deletion (phESC ${ }^{\Delta \mathrm{IG}}$ ) (Supplementary information, Figure S2C and S2F). Then we purified the haploid cells of these genetically modified phESC lines by FACS and generated bimaternal embryos by MII oocyte injection to investigate the gynogenetic development (Supplementary information, Figure S2D and S2G). A total of 624 phESC $^{\Delta 5 \mathrm{H} 19}$ embryos from 2 phESC ${ }^{\Delta \mathrm{H} 19}$ sublines, and 611 phESC $^{\Delta 15 H 19}$ embryos from 2 phESC $^{\Delta 15 H 19}$ sublines were transferred into pseudopreganant mothers. None developed to term (E19.5), though some embryos developed beyond E13.5 (Supplementary information, Table S1A). However, of the 568 phESC $^{\Delta \mathrm{IG}}$ embryos transplanted, $13(2.3 \%)$ full-term pups were recovered by cesarean section (Supplementary information, Table S1A). These phESC $^{\Delta \mathrm{IG}}$ pups were severely growth-retarded $(0.57 \pm$ $0.02 \mathrm{~g}, n=10$ ), with about $46 \%$ of normal body weight of WT pups $(1.25 \pm 0.04 \mathrm{~g}, n=12$; Figure $1 \mathrm{E}$ and $1 \mathrm{~F})$, and all died within several hours after birth. Comparison of global imprinting status between phESC ${ }^{\Delta I G}$ and WT pups showed that of 16 DMRs recovered by RRBS, 15 maternally methylated DMRs had similar methylation levels, but the H19-DMR was hypomethylated in the phESC $^{\Delta I G}$ pups (Figure $1 \mathrm{H}$ and Supplementary information, Figure S2L). Bisulfite sequencing analysis confirmed the normal methylation level of maternally methylated Igf2r-and Snrpn-DMRs, and hypomethylation of H19-DMR (Figure 1G).

Next, we deleted either the $5 \mathrm{~kb}$ or the $15 \mathrm{~kb}$ H19-Igf2 region in the phESC ${ }^{\Delta I \mathrm{G}}$ lines to generate double knockout (DKO) phESC lines (phESC ${ }^{\Delta \mathrm{IG} / \Delta 5 \mathrm{H} 19}$ or phESC ${ }^{\Delta \mathrm{IG} /}$ $\left.{ }^{\Delta 15 \mathrm{H} 19}\right)$. Of the 568 transplanted $\mathrm{phESC}^{\Delta \mathrm{IG} / \Delta 5 \mathrm{H} 19}$ embryos, $21(3.7 \%)$ live full-term pups were recovered (Supplementary information, Table S1A). The phESC ${ }^{\Delta \mathrm{IG} / \Delta 5 \mathrm{H} 19}$ pups had similar body weight $(0.57 \pm 0.01 \mathrm{~g}, n=13$; $46 \%$ of WT body weight) to the phESC ${ }^{\Delta I G}$ pups, and died soon after birth (Figure 1J). However, of the 475 transplanted phESC ${ }^{\mathrm{LI} / \Delta / 5 \mathrm{H} 19}$ embryos, $43(9.1 \%)$ live full-term pups were recovered, which had similar body weight $(1.34 \pm 0.03 \mathrm{~g}, n=30)$ to the WT pups (Figure 1I, $1 \mathrm{~J}$ and Supplementary information, Table S1A). Thirty-one $\operatorname{phESC}^{\Delta \mathrm{IG} / \Delta 15 \mathrm{H} 19}$ pups survived to adulthood, while the rest 12 died within 3 days after birth due to lack of nursing from the surrogate mothers (Supplementary information, Table S1A). Gene expression analysis showed that $\operatorname{Ig} f 2$ expression level was much lower in $\mathrm{phESC}^{\Delta \mathrm{IG} / \Delta 5 \mathrm{H} 19}$ pups, but was comparable between phESC ${ }^{\Delta \mathrm{IG} / \Delta 15 \mathrm{H} 19}$ and WT pups, indicating that $I g f 2$ expression was not activated by the $5 \mathrm{~kb}$ deletion of the H19-Igf2 locus (Figure 1K). To confirm whether the defect in Igf2 activation caused the growth retardation of the $\mathrm{phESC}^{\Delta \mathrm{IG} / \Delta 5 \mathrm{H} 19}$ pups, we knocked the exogenous $\operatorname{Ig} f 2$ gene into the endogenous $H 19$ gene locus in $\mathrm{phESCs}^{\Delta \mathrm{IG} / \Delta 5 \mathrm{H} 19}\left(\mathrm{phESCs}^{\Delta \mathrm{IG} / \Delta 5 \mathrm{H} 19 / \mathrm{lg} 2}\right)$ by replacing the "PGK-neo" cassette of the 5 kb-targeting

Figure 1 Birth of fertile bimaternal offspring following intracytoplasmic injection of phESCs. (A) Morphology of a live E13.5 bimaternal embryo. Scale bar, $2 \mathrm{~mm}$. (B) Heat map of the methylation level of ICRs in bimaternal and WT E13.5 fetuses. DMRs with significantly different methylation level between bimaternal and WT groups were marked with asterisk $(P<0.05)$. (C) Scatter plot comparison of transcriptomes of bimaternal and WT E13.5 fetuses. The raw FPKM for each gene was transformed to $\log _{2}$ value, and genes with more than 1 FPKM were shown. The red and blue points represent imprinted genes expressed from maternal and paternal alleles, respectively. Dashed lines depict 2-fold changes. The R2 was determined by Pearson's correlation. (D) Five imprinted genes with more than 2-fold changes in expression levels between bimaternal and WT embryos were shown. (E) A full-term bimaternal pup derived by oocyte injection of phESC ${ }^{\Delta l G}$. (F) The body and placenta weights of phESC ${ }^{\Delta \mid G}$ pups, showing severe retardation compared with WT pups. ${ }^{* * *} P<0.001$. (G) Bisulfite analysis of $H 19-$, Igf2r- and Snrpn-DMRs in phESC ${ }^{\Delta I G}$ pups. (H) RRBS analysis of whole brain of phESC ${ }^{\Delta l G}$ and WT pups. (I) Survived bimaternal pups derived by oocyte injection of phESC ${ }^{\Delta \mid G / \Delta 15 H 19}$. (J) The body and placenta weights of phESC ${ }^{\Delta / G / \Delta 5 H 19}$, phESC ${ }^{\Delta / G / \Delta 15 H 19}$ and WT pups. ${ }^{* * *} P<0.001$. (K) Gene expresson analysis showing reduced expression of lgf2 in phESC ${ }^{\Delta \mid G}$ and phESC ${ }^{\Delta / G / \Delta 5 H 19}$ pups, and its normal expression in phESC ${ }^{\Delta / G / \Delta 15 H 19}$ pups. (L) Survived bimaternal pups produced by oocyte injection of phESC ${ }^{\Delta \mathrm{G} / \Delta 5 \mathrm{H} 19 / \mathrm{gf} 2}$. (M) The body and placenta weights of phESC ${ }^{\Delta \mathrm{IG} / \Delta 5 \mathrm{H} 19 / \mathrm{gf} 2}$ and $\mathrm{WT}$ pups. (N) Body weight of the bimaternal mice 1-7 weeks after birth, showing $\sim 20 \%$ growth retardation compared with WT ones (WT female, red, $n=8$; phESC ${ }^{\Delta \mid G / \Delta 15 H 19}$ mice, blue, $n=8$; phESC ${ }^{\Delta \mid G / \Delta 5 H 19 / / g f 2}$ mice, black, $\left.n=8\right)$. The values represent the means $\pm S E M$. (O) An adult bimaternal mouse and its offspring. $(\mathbf{P})$ Corrected litter size of bimaternal mouse $(5.8 \pm 0.9, n=9)$ was comparable with the WT $(6.2 \pm 0.70, n=9)$. $P=0.70$. (Q) Summary of viability of bimaternal mice produced from phESCs carrying different imprinting modifications. $\mathrm{Y}$, viable; $\mathrm{N}$, non-viable. 
vector with an "EF1 $\alpha$-Igf2-2A-Rfp-IRES-neo" cassette (Supplementary information, Figure S2I). $\mathrm{phESCs}^{\Delta \mathrm{IG} / \Delta 5 \mathrm{H} 19 /}$ Igf2 carried the same $5 \mathrm{~kb}$ deletion of the H19-Igf2 locus as the phESCs ${ }^{\Delta I G / \Delta S H 19}$, but they expressed the exogenous $I g f 2$ and $R f p$ genes (Supplementary information, Figure $\mathrm{S} 2 \mathrm{~J}$ and $\mathrm{S} 2 \mathrm{~K}$ ). We then injected the haploid phESCs ${ }^{\Delta \mathrm{IG} /}$ ${ }_{\Delta 5 \mathrm{H} 19 / \mathrm{Igf2}}$ into MII oocytes and transplanted the embryos into the psudopregnant mice to test their developmental ability (Supplementary information, Figure S2L). Of the 450 transplanted embryos, 32 live full-term pups were generated (Figure 1L and Supplementary information, Table S1A). Interestingly, the phESC $\mathrm{C}^{\Delta \mathrm{IG} / \Delta 5 \mathrm{H} 19 / \mathrm{Igf} 2}$ pups (1.55 $\pm 0.04 \mathrm{~g}, n=24$ ) had similar size and body weight to the WT pups (Figure 1M). Twenty-three pups survived to adulthood, and the rest 9 pups died due to lack of nursing from the surrogate mothers (Supplementary information, Table S1A).

We analyzed the postnatal growth of the survived bimaternal mice by recording their body weight every week. Though born with normal body weight, the bimaternal mice were consistently $\sim 20 \%$ lighter than the WT controls since the first week (Figure 1N). Except for the growth retardation, no obvious abnormalities were observed in the bimaternal mice. The female bimaternal mice were mated with WT males to examine their fertility. A total of 29 offspring were delivered in 9 litters (Figure 1O). Four neonatal pups carrying an $I G$-DMRknockout allele died soon after birth, and the rest 25 mice grew into adults (Supplementary information, Table S1B), consistent with a previous finding that mice with a maternally inherited $I G$-DMR deletion died at later gestational stages [12]. According to the Mendelian ratio, the real litter size of bimaternal mice was calculated as 5.8 \pm 0.9 , comparable to the litter size of WT females $(6.2 \pm$ $0.7)$, indicating that the bimaternal mice had normal fertility (Figure 1P).

In summary, here we show that the MII oocyte-derived phESCs can efficiently produce bimaternal mice through injection into WT MII oocytes after proper imprinting modifications. Compared to previous reports that used non-growing oocytes to achieve gynogenetic development [4], our approach provides a more robust platform to study the developmental roles of genomic imprinting. For example, we showed that the $I G$-DMR deletion, but not H19-DMR deletion, was suffcient to produce fullterm bimaternal pups, indicating that the Dlk1-Dio3 region has a more profound effect on fetal growth. Though both the $15 \mathrm{~kb}$ and $5 \mathrm{~kb}$ regions contained the ICR of the H19-Igf2 cluster, only the $15 \mathrm{~kb}$ deletion could reactivate Igf 2 expression and support the adult development of bimaternal mice, suggesting that the H19-Igf2 imprinting was regulated not only by the methylation of ICR, but also by the relative distance and spatial organizations of different genomic elements in this region (Figure 1Q). In general, we have established a convenient and efficient approach to generate bimaternal mammals from imprinting-modified phESCs, which can provide a novel platform for genomic imprinting studies, and may also shed new light on mammalian reproduction. Given the successful establishment of phESCs in the mouse, rat and monkey, we expect that our approach can also be applied in other mammalian species to produce bimaternal animals.

\section{Acknowledgments}

This work was supported by the National Basic Research Program of China (973 Program; 2012CBA01300, 2012CB966302 and 2014CB964801), the National Natural Science Foundation of China (91319308 and 31422038), and the "Strategic Priority Research Program" of the Chinese Academy of Sciences (XDA01020101). We thank Ting Li, Qing Meng, and Shi-Wen Li for their help with FACS and confocal laser scanning microscopy.

\footnotetext{
Zhikun $\mathrm{Li}^{1,2, *}$, Haifeng Wan ${ }^{1, *}$, Guihai Feng ${ }^{1,3, *}$, Leyun Wang ${ }^{1,4, *}$, Zhengquan $\mathrm{He}^{1}$, Yukai Wang ${ }^{1}$, Xiu-Jie Wang ${ }^{3}$, Wei $\mathrm{Li}^{1}$, Qi Zhou ${ }^{1}$, Baoyang $\mathrm{Hu}^{1}$

${ }^{I}$ State Key Laboratory of Stem cell and Reproductive Biology, Institute of Zoology, Chinese Academy of Sciences, Beijing 100101, China; ${ }^{2}$ University of Chinese Academy of Sciences, Beijing 100049, China; ${ }^{3}$ Key Laboratory of Genetic Network Biology, Institute of Genetics and Developmental Biology, Chinese Academy of Sciences, Beijing 100101, China; ${ }^{4}$ College of Life Science, Northeast Agricultural University of China, Harbin, Heilongiiang 150030, China

*These four authors contributed equally to this work.

Correspondence: Baoyang $\mathrm{Hu}^{\mathrm{a}}$, Qi Zhou ${ }^{\mathrm{b}}$, Wei $\mathrm{Li}^{\mathrm{c}}$

a'E-mail: byhu@ioz.ac.cn

bE-mail: qzhou@ioz.ac.cn

cE-mail: liwei@ioz.ac.cn
}

\section{References}

1 Surani MA, Barton SC, Norris ML. Nature 1984; 308:548-550.

2 Surani MA, Barton SC, Norris ML. Nature 1987; 326:395-397.

3 Mann JR, Lovellbadge RH. Nature 1984; 310:66-67.

4 Kono T, Obata Y, Wu QL, et al. Nature 2004; 428:860-864.

5 Leeb M, Wutz A. Nature 2011; 479:131-134.

6 Yang H, Shi L, Wang BA, et al. Cell 2012; 149:605-617.

7 Li W, Shuai L, Wan H, et al. Nature 2012; 490:407-411.

8 Wan H, He Z, Dong M, et al. Cell Res 2013; 23:1330-1333.

9 Bell AC, Felsenfeld G. Nature 2000; 405:482-485.

10 Hark AT, Schoenherr CJ, Katz DJ, et al. Nature 2000; 405:486-489.

11 Leighton PA, Ingram RS, Eggenschwiler J, et al. Nature 1995; 375:3439.

12 Lin SP, Youngson N, Takada S, et al. Nat Genet 2003; 35:97-102.

13 Webber AL, Ingram RS, Levorse JM, et al. Nature 1998; 391:711-715.

(Supplementary information is linked to the online version of the paper on the Cell Research website.) 